\title{
The CMS Muon System
}

\author{
Anna Colaleo* \\ On behalf of CMS Collaboration \\ CERN and INFN-Bari \\ Via Amendola 173, Bari Italy \\ E-mail: Anna.Colaleo@cern.ch
}

\begin{abstract}
The CMS muon system was designed to serve both for the offline muon track reconstruction and for triggering purposes. The high redundancy and robustness of the system are ensured by three complementary subsystems: Drift Tube chambers in the barrel, Cathode Strip Chambers in the endcap and Resistive Plate Chambers placed both in the barrel and in the endcaps. The installation of the muon stations in the above-ground site of CMS is underway and it will be completed in 2006. In this paper the muon system, its construction status and commissioning plans will be reviewed.
\end{abstract}

European Physical Society

HEP2005 International Europhysics Conference on High Energy Physics EPS (July $21^{\text {st }}-27^{\text {th }}$ 2005) in Lisboa, Portugal

\footnotetext{
* Speaker 


\section{Introduction}

Muons with high transverse momenta $\left(\mathrm{p}_{\mathrm{t}}\right)$ will provide a clean signature for many of the interesting processes which will be studied at the LHC. The CMS Muon Detector [1] has been designed to identify and reconstruct muons with a momentum resolution $\Delta \mathrm{p}_{\mathrm{t}} / \mathrm{p}_{\mathrm{t}}$ in the range of $8-15 \%$ at $10 \mathrm{GeV} / \mathrm{c}$ and $20-40 \%$ at $1 \mathrm{TeV} / \mathrm{c}$ and trigger on them with well defined $\mathrm{p}_{\mathrm{t}}$ threshold up to $|\eta|=2.4$. The muon system relies on the solenoidal magnetic field in the iron return yoke to estimate the muon $\mathrm{p}_{\mathrm{t}}$ from the track bending. Drift Tube (DT) in barrel and Cathod Strip Chambers (CSC) in the end caps will provide a very good position resolution and trigger efficiency, whereas a dedicated trigger system is realized by Resistive Plate Chambers (RPC) in both regions. The choice of the detector technology has been driven by the very large surface to be covered and by the background radiation environment. The L1 muon trigger [2] makes use of all three muon detectors DT, CSC and RPC, each one with its own trigger logic. Up to four best (highest $\mathrm{p}_{\mathrm{T}}$ and quality) muon candidates from each system are selected and sent to the Global Muon Trigger [2].

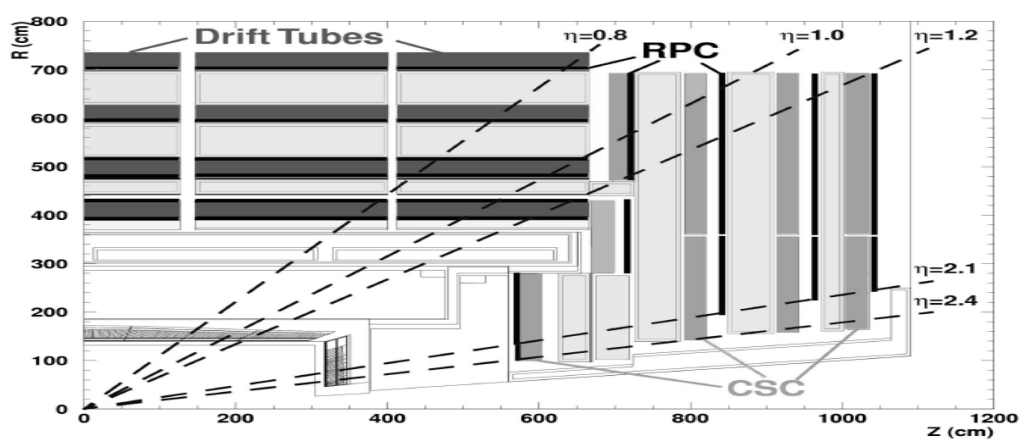

Figure 1 A longitudinal view of one quadrant of the CMS detector.

\subsection{Cathod Strip Chambers}

In the End Cap region CSC chambers will provide precise time and position measurement. CSC's are trapezoidal shaped chambers consisting of six layers of multi-wire proportional chamber. A layer is defined by two parallel cathode planes with a gas gap filled with $30 \% \mathrm{Ar}$, $50 \% \mathrm{CO}_{2}$, and $20 \% \mathrm{CF}_{4}$. One cathode is segmented into radial strips running perpendicular to the anode wires and measuring the $\phi$ coordinate. The avalanche produced by a crossing charged particle is collected by the anode wires, and the image charge is collected in several adjacent strips. The wire signal gives a fast information with a rough spatial resolution $\mathrm{O}(\mathrm{mm})$. A precise spatial measurement $(\sigma(\mathrm{r}-\phi) \sim 100-240 \mu \mathrm{m})$ is achieved by determining the center of gravity of the charge distribution induced on the cathode strips. The CSC detectors use the multi-layer structure for self triggering. A Local Charged Track (LCT) is built from the spatial and time correlation of the hits in the two projections. The anode signals are used to identify the bunch crossing (bx) with a multi-layer time coincidence in the LCT pattern. 
Mechanically, the detectors were assembled at Fermilab, PNPI (St. Petersburg), and IHEP (Beijing). Assembly of chambers and detector performance validation took place at UF, UCLA, PNPI, and IHEP — at so-called FAST (Final Assembly and System Tests) sites.

\subsection{Drift Tube chambers}

The barrel region is instrumented with DT chambers. A muon chamber is built up from three so-called super-layers with four layers of half-a-cell width staggered drift tubes (Fig.2). Eight layers in a chamber measure the $(\mathrm{r}, \phi)$-coordinate while only four layers measure the $(\mathrm{r}$, z)-coordinate. The gas is a mixture of $85 \% \mathrm{Ar}$ and $15 \% \mathrm{CO}_{2}$. The important features of these chambers are the self-triggering and the bunch crossing identification capabilities. The Bunch and Trigger Identification (BTI) electronic combines the drift time measured in three consecutive layers, applying a mean timer technique. The excellent linearity of the time-todistance relationship of the cell allows a time resolution of about 4 ns [3]. At the same time, track segments of aligned hits are built. The multi-layer structure of the chambers improves the spatial resolution of the single cell, which reaches the needed $100 \mu \mathrm{m}$. The DT trigger electronic assigns the correct bx to a muon track with $>99 \%$ efficiency.

Chambers are produced and tested at 4 sites: Aachen, CIEMAT, Legnaro and Torino. The production is on schedule and the plan is to complete chamber production by February 2005.

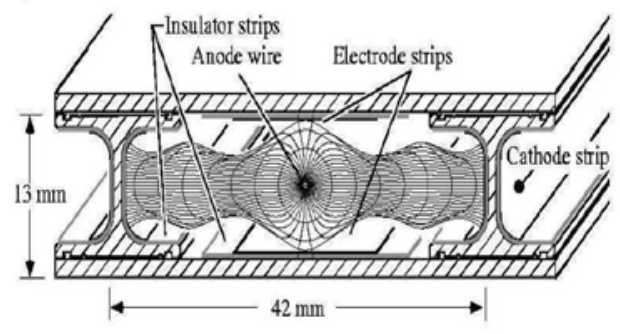

Figure 2 Schematic view of a DT cell.

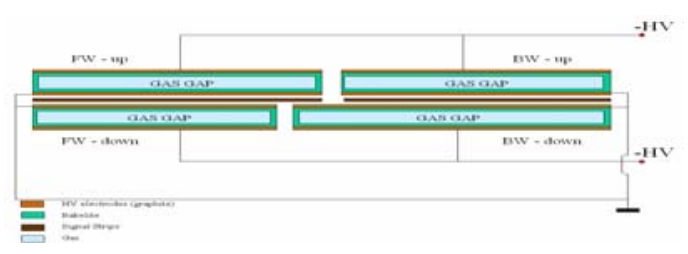

Figure 3. Schematic view of a double gap RPC

\subsection{Resistive Plate Chambers}

RPC chambers are installed in both the barrel and the endcaps as dedicated trigger detectors. They guarantee a precise bx assignment of the muon tracks thanks to their fast response and good time resolution $\left(\sigma_{\mathrm{t}}<1.5 \mathrm{~ns}\right.$ [4]). The barrel muon stations are equipped with two RPC layers for the two innermost stations and with one layer for each outer station (total 6 RPC layers). The end caps are instrumented with one RPC layer per station (total 4 RPC layers).

An RPC gap is made by two parallel bakelite plates $\left(1-210^{10} \Omega \cdot \mathrm{cm}\right.$ resistivity) placed at a distance of $2 \mathrm{~mm}$ and filled with a gas mixture of $96.2 \% \mathrm{C}_{2} \mathrm{H}_{2} \mathrm{~F}_{4}, 3.5 \% \mathrm{i}_{-} \mathrm{C}_{4} \mathrm{H}_{10}$ and $0.3 \%$ of $\mathrm{SF}_{6}$. In CMS double-gap RPC's are used (Fig.3), with two gas gaps read out by a single set of copper strips in the middle in order to increases the induced signal. The chambers are operated in avalanche mode to ensure proper operation at high background rates of up to $10 \mathrm{kHz} / \mathrm{cm}^{2}$. The RPC system is used also to identify and measure muon tracks at the Level 1 trigger with a 
pattern comparator logic. Muons are reconstructed from correlated patterns of hits in the different stations.

RPC barrel chambers are assembled at the HiTec and General Tecnica factories and in the Bari and Sofia laboratories. They are characterized with cosmic rays at the station sites of Bari, Pavia and Sofia. Production will be completed in March 2006. The endcap gas gaps are produced in Korea whereas the chambers are assembled and tested in Pakistan and at CERN under China responsibility.

\section{Installation and commissioning plans}

All chambers are shipped at ISR CERN area where they undertake the final dressing and integrity checks before to be certified as ready for installation with cosmic rays data taking and long term test. DT alignment is also performed. DT and RPC chambers are mechanically coupled and then sent at SX5, the above-ground site of CMS where they are installed inside the iron.
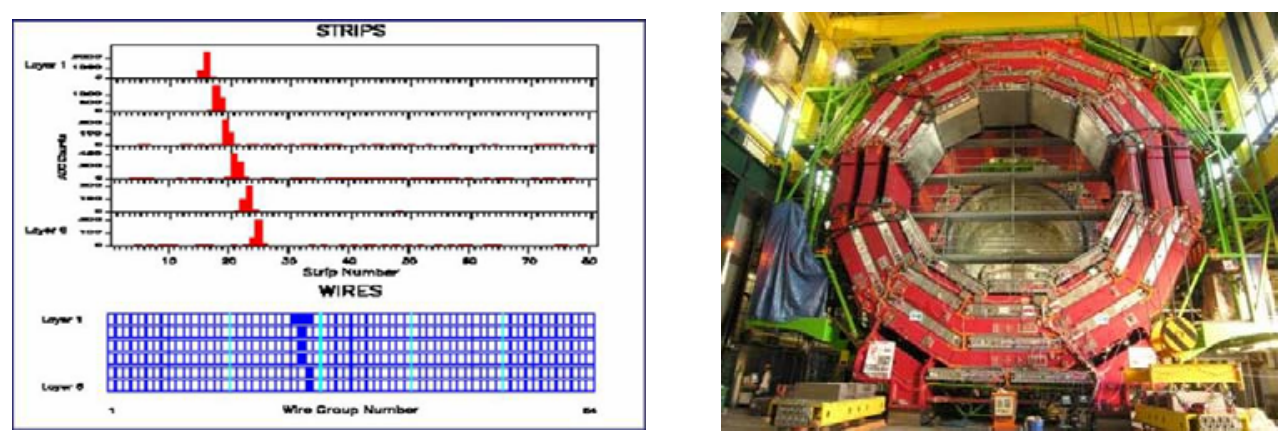

Figure 4. On left side a cosmic ray observed with CSC chambers during the commissioning. On right side the barrel muon stations installed in the wheel at SX5.

After installation the single chamber functionalities are checked with cosmic rays data taking in self-trigger mode. As for July $200550 \%$ of CSC chambers have been installed, two wheels of the barrel system has been installed and commissioning is ongoing (Fig.4). RPC endcap chambers installation starts in October 2005.

The first CMS magnet test and the "cosmic challenge" test in spring 2006 in the surface building SX5 will allow the muon system to test the alignment system, check deformations, component integration, establish timing and calibration procedures with the CMS Data Acquisition System and Detector Control System.

\section{References}

[1] CMS Collaboration, The Muon Project, Technical Design Report CERN/LHCC 97-32,

[2] CMS Collaboration, CMS Trigger Technical Design Report, Volume 1: The Trigger Systems. CERN/LHCC (2000-38).

[3] M. Cerrada et al., CMS-NOTE 2003/007 (2003).

[4] M. Abbrescia et al., Nucl. Instr. And Meth. A 456 (2000) 103-108. 